\title{
Dementia and Parkinsonism-a Rare Presentation of Intracranial Dural Arteriovenous Fistulae
}

\author{
Manoj Gopinath, MD, Chinmay Nagesh, MD, Santhosh K, MD, PDCC, Jayadevan ER, MD, DM
}

Intracranial dural arteriovenous fistulae (DAVF) are acquired fistulous communications between dural arterial branches and dural venous sinuses or cortical veins with the nidus located within the leaflets of the duramater. Dementia and Parkinsonism are amongst the rarest of clinical presentations in DAVFs and are important to diagnose early, being treatable with timely intervention. We present an interesting case of a patient who presented with rapidly progressive dementia and features of parkinsonism who was diagnosed to have extensive DAVF and made remarkable recovery after embolization of the fistulae.

Key Words : Dural arteriovenous fistula; Embolization; Dementia; Parkinsonism

Intracranial Dural Arteriovenous Fistulae (DAVF) are acquired fistulous communications between dural arterial branches and dural venous sinuses or cortical veins with the nidus located within the leaflets of the duramater. They are classified as benign or aggressive based primarily on their venous drainage and location. DAVFs are known to have myriad clinical presentations of which headache and tinnitus are considered the commonest. Dementia and Parkinsonism are amongst the rarest of clinical presentations in DAVFs and are important to diagnose early, being treatable with timely intervention. We present an interesting case of a patient

All authors: Department of Imaging Sciences and Interventional Radiology, Sree Chitra Tirunal Institute for Medical Sciences and Technology, Trivandrum, Kerala. India

Received March 13, 2017; Revised August 7, 2017; Accepted August 9, 2017.

Correspondence to: Manoj Gopinath, MD, Department of Imaging Sciences and Interventional Radiology, Sree Chitra Tirunal Institute for Medical Sciences and Technology, Trivandrum, Kerala, India Tel. +919400408866 E-mail: drmanojg@sctimst.ac.in

This is an Open Access article distributed under the terms of the Creative Commons Attribution Non-Commercial License (http://creativecommons.org/licenses/by-nc/3.0) which permits unrestricted non-commercial use, distribution, and reproduction in any medium, provided the original work is properly cited. who presented with rapidly progressive dementia and features of parkinsonism who was diagnosed to have extensive DAVF and made remarkable recovery after embolization of the fistulae.

\section{CASE REPORT}

45 year old female patient was brought to our hospital with 4 months history of loss of interest in routine daily activities and inactiveness. The symptoms were insidious in onset with rapid progression over 4 months and sudden worsening in 1 week prior to presentation. There was no history of emotional lability, hallucinations or memory disturbances. There was no history of falls, and no sensory or motor deficits.

Her general examination and vital signs were unremarkable. Neurological examination revealed severe apathy and impaired attention span and needed repeated coaxing to cooperate. Speech was hypophonic. She could follow single commands. Naming was normal but reading and writing were impaired. Mini Mental State Examination (MMSE) score was 18/30. Bilateral Papilledema and reduced abduction of both eyes was noted. The other cranial nerves were normal. There was evidence of rigidity in 


\section{Chinmay Nagesh, et al.}

all four limbs and bradykinesia was present. Power was normal and all reflexes appeared exaggerated. Plantars were extensor. Gait was slow with narrow base and slow shuffling associated with body bradykinesia. There were no signs of meningeal irritation.

Her baseline hematological and biochemical investigations were normal apart from peripheral blood smear which revealed microcytic hypochromic picture. Magnetic Resonance Imaging (MRI) brain was done (Fig. 1) revealing diffuse deep white matter and periventricular T2 and FLAIR hyperintensities bilaterally which was followed by Digital Subtraction Angiography (DSA) (Fig. 2), which revealed torcular and Superior sagittal sinus DAVFs with arterial feeders from Internal Carotid Artery (ICA) via marginal tentorial artery and from External Carotid artery (ECA) through occipital and middle meningeal artery branches.

In view of the presentation with rapidly progressive dementia with parkinsonism and clinical findings of apathy, cerebral dysfunction, raised ICP and parkinsonism with features of pyramidal dysfunction all likely due to venous congestion secondary to DAVF demonstrated in DSA, she underwent endovascular intervention in the form of Squid embolization of the fistula. The arterial feeders to the right Transverse and sigmoid sinuses and torcula as well as the superior sagittal sinus were embolized.

Post procedure her sensorium improved slowly. She was discharged with advise to follow up. Features of parkinsonism persisted for a few weeks for which Levodopa + Carbidopa was initiated. Over the next 6 months, patient made good progress, became asymptomatic without any residual features of parkinsonism and medication were stopped and she was back to her routine daily activities. A check DSA done after 6 months revealed a residual/recurrent DAVF at the torcula (Fig. 3) for which she underwent a second embolization procedure a few weeks later (Fig. 4). She continued to remain on follow up. Presently she is asymptomatic without any residual neurodeficit and continuing with all her routine daily activities without hindrance. She underwent an MRI scan during follow up which revealed significant resolution of the white matter hyperintensities which were previously seen (Fig. 5). She is planned to undergo another check DSA a year later to reassess the status.

\section{DISCUSSION}

DAVF are generally accepted to be acquired fistulous communications between dural arterial branches and dural venous sinuses or cortical veins with the nidus located within the leaflets of the duramater. They account for $10-15 \%$ of the cerebrovascular malformations [1]. Prior cerebral venous sinus thrombosis is implicated in formation of DAVF and transverse and sigmoid sinus location is described as the commonest

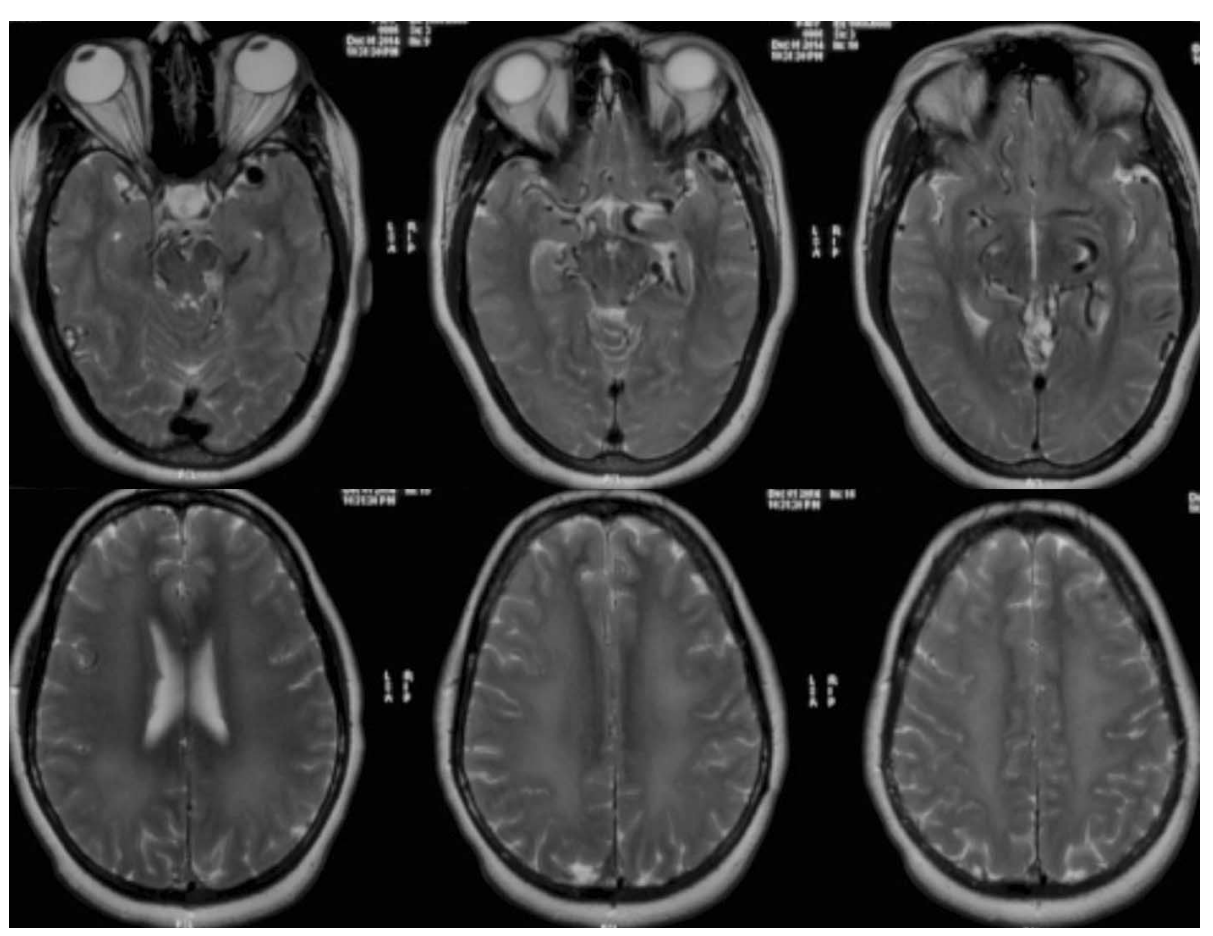

Fig. 1. Axial T2-weighted images reveal tortuous flow voids in cisterns and sylvian fissure with prominent enlarged torcula and bilateral, diffuse white matter hyperintensities. 

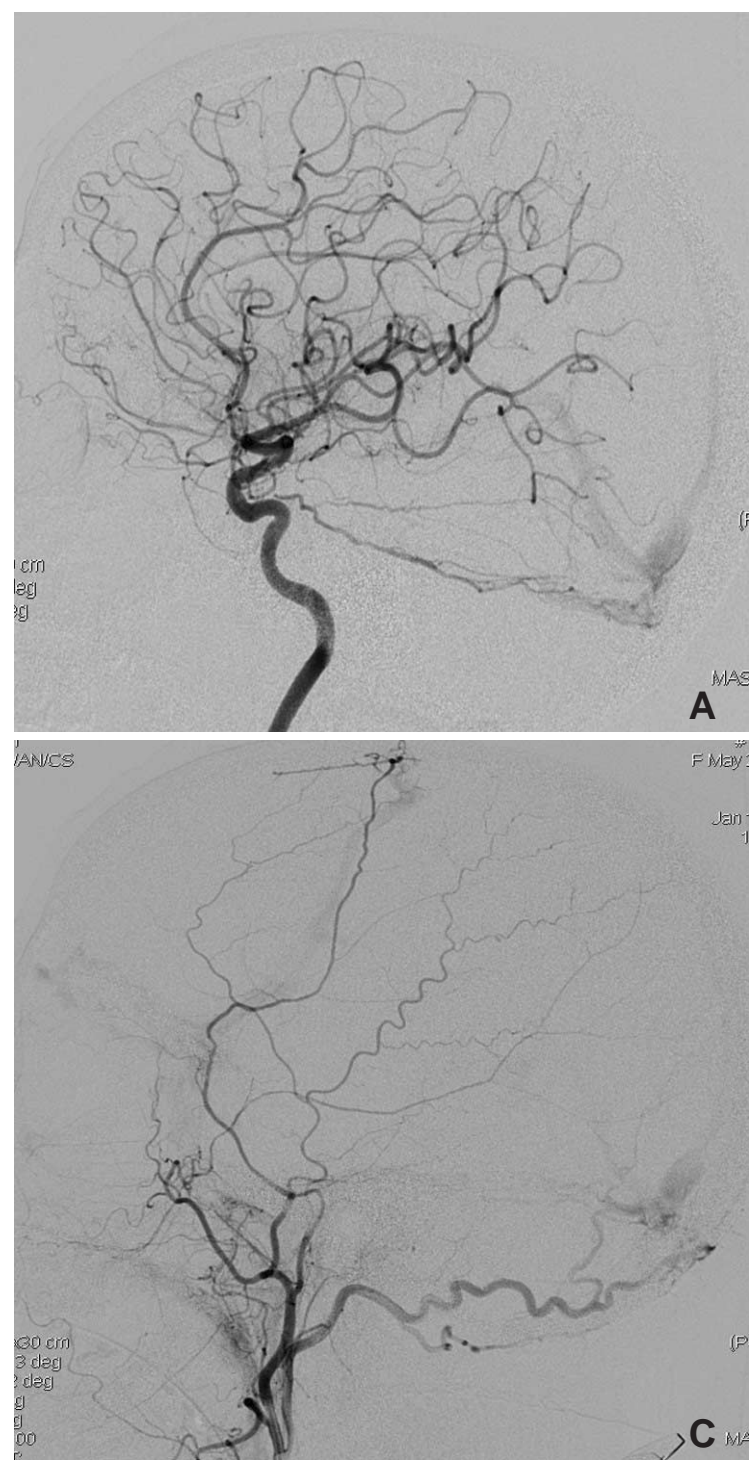

comprising $60 \%$ of the cases [2]. Dementia and Parkinsonism are uncommon presentations in DAVF with only a few cases reported in literature. The exact pathophysiology is still not fully known with various hypothesis put forth by different authors. Matsuda et al described a series of cases with dementia and parkinsonism as presenting features and they compared the pathophysiology with vascular parkinsonism and highlighted the presence of white matter hyperintensity in their cases [3]. Lee et al postulated that basal ganglia dysfunction secondary to impaired venous outflow is the cause for such a presentation [4]. Hang et al used dopamine transporter imaging to demonstrate presynaptic dopaminergic nerve terminal loss in a group of patients with parkinsonism as clinical presentation and also described the reversibility of the condition after occlusion of the fistula [5]. It seems

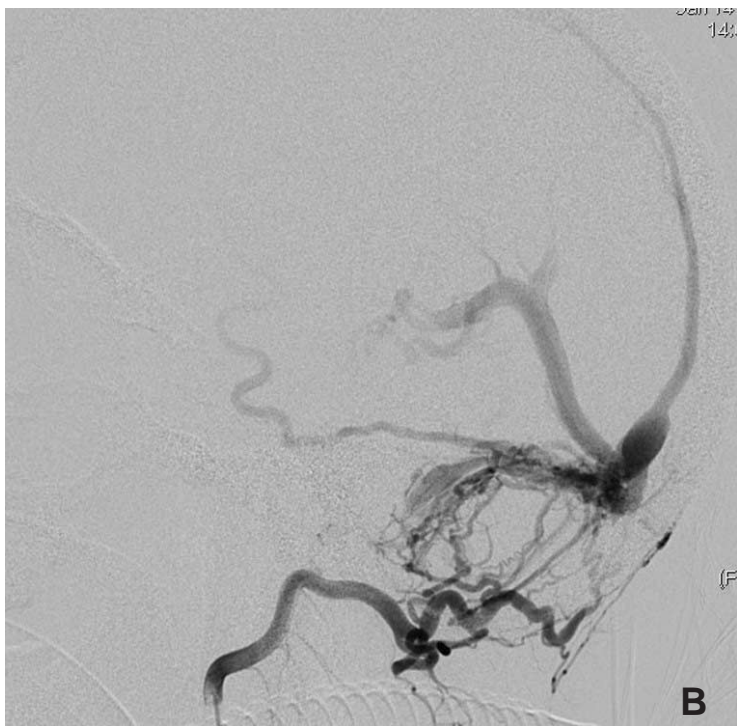

Fig. 2. DSA images reveal torcular and superior sagittal sinus DAVFs with arterial feeders from $(\mathbf{A})$ internal carotid artery via marginal tentorial artery, from external carotid artery via (B) occipital and (C) middle meningeal branches.

likely that those DAVF with cortical venous reflux from the fistula will eventually cause increased venous pressure intracranially. This congestion would lead to compromised venous drainage from normal parenchyma with ischemic sequelae over a period of time [6]. It is at this point that patients would present with encephalopathy and dementia. Extrapyramidal features are also likely due to compromised venous outflow from basal ganglia and may not always be associated with abnormal imaging findings.

DAVF present with hemorrhagic and non hemorrhagic neurological manifestations depending on the site of fistula and the pattern of venous drainage. Dementia and parkinsonism are both uncommon presentations of this disease entity, comprising 3-12\% of cases [7]. Patients often go undiagnosed for a long time leading to further worsening and irreversible neurological 


\section{Chinmay Nagesh, et al.}

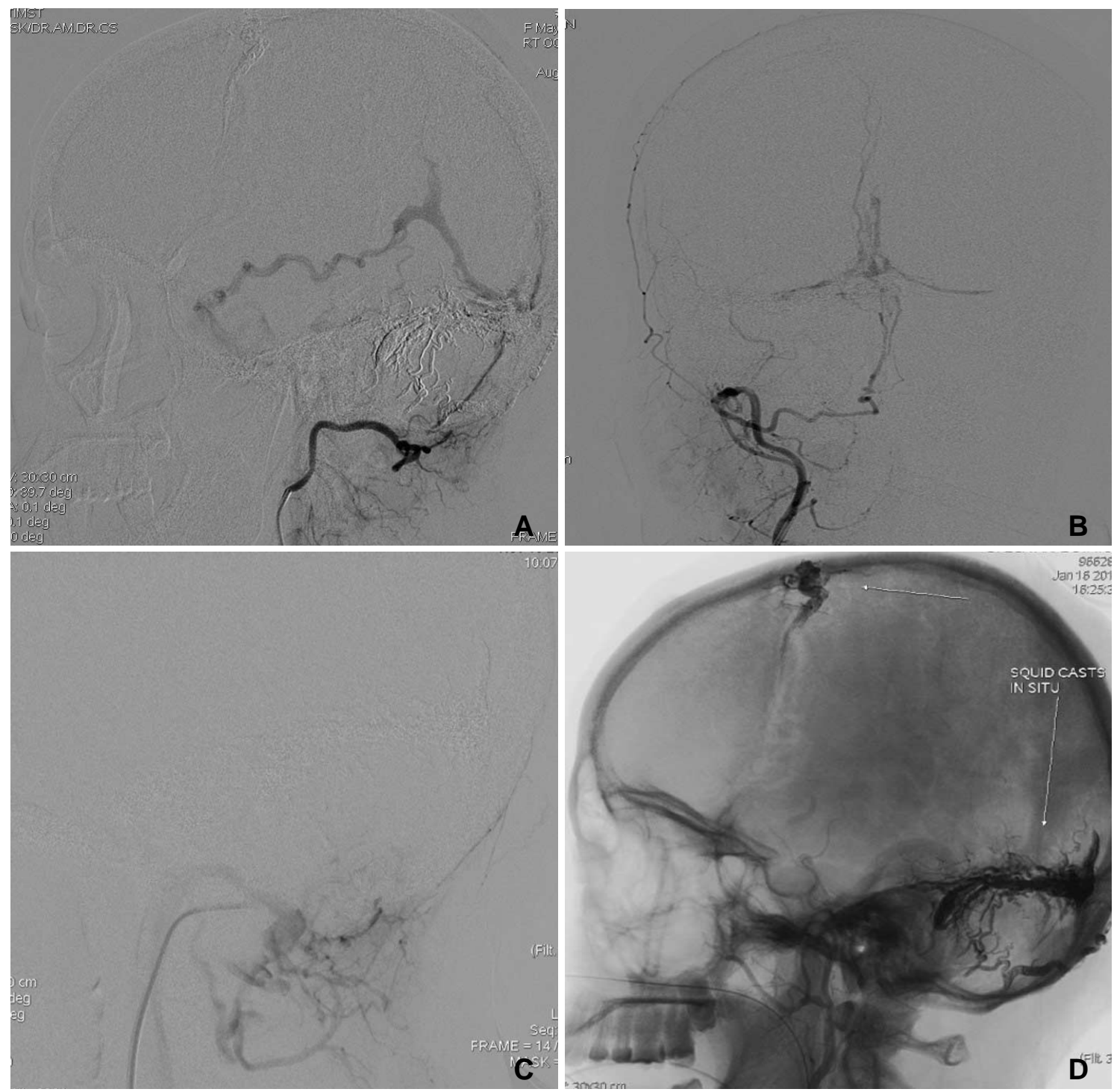

Fig. 3. Follow-up angiograms (A, B) show residual torcular DAVF with arterial supply from occipital artery. (C) Post-embolization angiogram shows obliteration of shunt at torcula. (D) Post-embolization status with squid casts in situ in superior sagittal sinus and torcula.
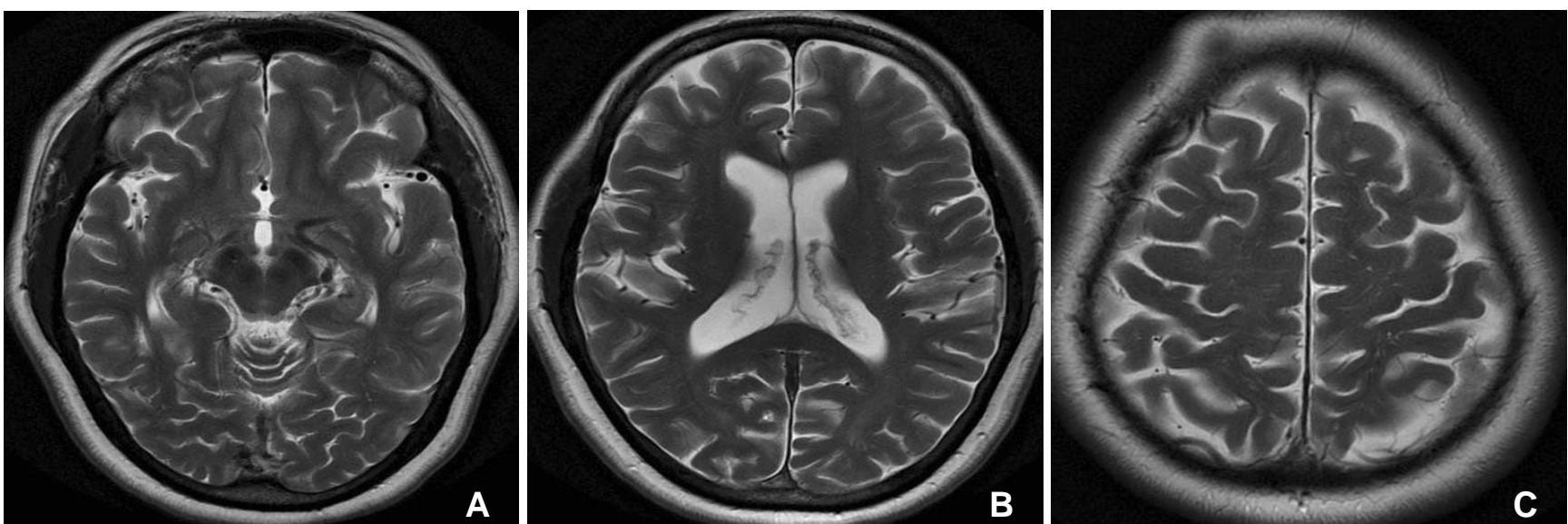

Fig. 4. Follow-up, axial T2-weighted images (A-C) show significant resolution of T2 white matter hyperintensities that were seen in the initial pretreatment MRI. 


\section{Dementia and Parkinsonism due to Dural AV Fistula}

deficits. High index of clinical suspicion, coupled with appropriate imaging is vital for early diagnosis of this condition $[8,9]$.

Non Contrast Computed Tomography (NCCT) is usually the first modality to be utilized and rarely shows any diagnostic findings but subtle findings in form of vascular calcification may be evident within the venous channels and dural sinus walls. MRI may reveal white matter hyperintensity and dilated flow voids of venous channels. Susceptibility Weighted Imaging (SWI) sequence in MRI has improved the diagnostic value significantly by revealing hyperintensity within the sinus or vein, indicating the site of fistula. Time of Flight (TOF) MRA may reveal the hypertrophied feeding arteries adjacent to the fistula. Angiography remains the gold standard for diagnosis and provides the requisite details for planning definite endovascular treatment. Functional imaging in form of PET/SPECT with dopamine transporter agents may be helpful in delineating the site of involvement better but are still not widely in use presently [10]. Clinical follow up is critical for long term management since recurrences are not uncommon and may need a second intervention at a later date which was well illustrated in our case.

DAVF is a potentially treatable cause of dementia and parkinsonism but diagnosis is often delayed due to the non specific nature of presentation. High clinical index of suspicion is warranted along with detailed clinical examination and appropriate imaging so that the diagnosis is made early and definite intervention planned.

\section{CONCLUSION}

Dementia and parkinsonism are uncommon presentations in DAVF. This is attributed to raised ICP and venous congestion within the cerebral parenchyma which if undiagnosed and untreated deteriorates to irreversible neuronal damage and permanent deficits. The importance of early diagnosis and definitive management lies in the fact that DAVF are among the few treatable causes of dementia and parkinsonism. The present case demonstrates the typical presentation in such a scenario and the gratifying outcome of early intervention and close follow up.

\section{References}

1. Lasjaunias P, Chiu M, ter Brugge K, Tolia A, Hurth M, Bernstein M. Neurological manifestations of intracranial dural arteriovenous malformations. J Neurosurg 1986;64:724-730

2. Awad IA, Little JR, Akarawi WP, Ahl J. Intracranial dural Arteriovenous malformations: factors predisposing to an aggressive neurological course. J Neurosurg 1990;72:839-850

3. Matsuda S, Waragai M, Shinotoh H, Takahashi N, Takagi K, Hattori T. Intracranial dural arteriovenous fistula (DAVF) presenting progressive dementia and parkinsonism. J Neurol Sci 1999; 165:43-47

4. Lee PH, Lee JS, Shin DH, Kim BM, Huh K. Parkinsonism as an initial manifestation of dural arteriovenous fistula. Eur J Neurol 2005;12:403-406

5. Kim HR, Lee JY, Kim YK, Park H, Kim HJ, Son YJ, et al. Dural Arteriovenous Fistula-Associated Reversible Parkinsonism with Presynaptic Dopaminergic Loss. J Mov Disord 2015;8:141-143

6. Ma C, Lu Q, Shi W, Su Z, Zhao Y, Li C, et al. Diagnosis and treatment of a dural arteriovenous fistula presenting with progressive parkinsonism and dementia: a case report and literature review. Exp Ther Med 2015;9:523-526

7. Hurst RW, Bagley LJ, Galetta S, Glosser G, Lieberman AP, Trojanowski J, et al. Dementia resulting from dural Arteriovenous fistulas: the pathologic findings of venous hypertensive encephalopathy. AJNR Am J Neuroradiol 1998;19:1267-1273

8. Netravathi M, Pal PK, Bharath RD, Ravishankar S. Intracranial dural arteriovenous fistula presenting as parkinsonism and cognitive dysfunction. J Clin Neurosci 2011;18:138-140

9. Enofe I, Thacker I, Shamim S. Dural arteriovenous fistula as a treatable dementia. Proc (Bayl Univ Med Cent) 2017;30:215-217

10. Luo Y, Qi J, Cen Z, Hu H, Jiang B, Luo W. Two cases of dural arteriovenous fistula presenting with parkinsonism and progressive cognitive dysfunction. J Neurol Sci 2014;343:211-214 